\title{
Relever ensemble les nouveaux défis de la lutte contre les cancers
}

\author{
Facing the New Challenges of Cancer Together
}

\author{
Marisol Touraine - Ministre des Affaires sociales et de la Santé \\ Geneviève Fioraso - Secrétaire d'État à l'Enseignement supérieur et à la Recherche
}

(C) Springer-Verlag France 2014

La mobilisation des communautés scientifiques et médicales a permis de réaliser de grandes avancées dans le domaine de la cancérologie. Si le nombre de nouveaux cas de cancers n'a cessé d'augmenter, le taux de mortalité par cancer baisse lui régulièrement depuis près d'une trentaine d'années en France. Mais nous le savons, malgré ces progrès thérapeutiques, les cancers demeurent la première cause de décès dans notre pays.

Deux Plans cancer ont déjà été mis en œuvre, pour les périodes 2003-2007 et 2009-2013. Ils ont permis de structurer l'organisation de la cancérologie, d'améliorer globalement la qualité des soins et des pratiques et, ainsi, la prise en charge des personnes malades. Ils ont également favorisé le déploiement de la recherche clinique et l'engagement de la France dans la médecine personnalisée à travers l'accès d'un nombre croissant de malades à des thérapies ciblant les caractéristiques génétiques de leur tumeur.

L'effort doit se poursuivre et s'intensifier pour répondre aux besoins de nos concitoyens - ils sont près de trois millions aujourd'hui - qui vivent avec et après un cancer, et pour soutenir les professionnels de la santé et de la recherche dans leur volonté de les guérir et d'améliorer leur qualité de vie. C'est l'objectif de ce troisième Plan cancer, qui a également été voulu par le Président de la République comme un véritable plan de lutte contre les inégalités face à la maladie. Il a pour ambition de donner à tous les mêmes chances de guérison partout en France, mais aussi de permettre à chacun de réduire son risque de cancer grâce à des actions de prévention adaptées.

Tout comme l'ensemble de notre système de santé, la politique de lutte contre les cancers doit répondre à ce double enjeu : s'engager réellement dans une approche préventive et ce, sans que celle-ci ne profite qu'aux catégories sociales les plus aisées comme cela a trop souvent été le cas jusqu'à présent. Le plan national de réduction du tabagisme sera un outil majeur pour faire baisser l'incidence des pathologies cancéreuses.

Il nous faut également continuer à faire évoluer l'offre de soins, pour qu'elle intègre le plus rapidement possible les innovations médicales et technologiques issues des travaux de recherche, au bénéfice de tous les patients. L'innovation en cancérologie, c'est aussi l'organisation de parcours de soins plus fluides, qui anticipent et prennent en compte les besoins de chaque patient, lui permettant de préserver au mieux sa vie personnelle et professionnelle le cas échéant.

Les progrès face aux cancers ne seront ainsi possibles que par un décloisonnement et des collaborations fortes : entre la recherche biomédicale, la recherche en sciences humaines et sociales et les soins; entre les professionnels hospitaliers et les professionnels de premier recours ; entre les acteurs de la santé et les intervenants sociaux. Sans oublier la concertation avec les premiers concernés, les personnes malades et leurs proches, les usagers du système de santé, les citoyens dont les préoccupations doivent être partagées avec les professionnels, dans une démarche de démocratie sanitaire renforcée sur tous les plans, de la prise en charge individuelle aux orientations des politiques publiques de santé.

C'est un choix décisif que fait notre pays, dans le contexte économique que nous connaissons, que de placer la lutte contre les cancers au rang de priorité nationale et de l'inscrire en tant que telle au cœur de nos stratégies nationales de santé et de recherche. Dans cette lutte contre la maladie, nous savons pouvoir compter sur l'engagement de toute la communauté du cancer, chercheurs, professionnels du secteur sanitaire et social, acteurs associatifs et bénévoles, rassemblés autour de l'Institut national du cancer. Soyez assurés que le nôtre est à la hauteur des défis que nous avons à relever collectivement. 